\title{
A Study on Development of OTIP System using QR Code based on Smartphone
}

\author{
Dae-Jea Cho and Mi-Young Bae \\ Dept. Of Multimedia Engineering, Andong National University, South KOREA \\ djcho@anu.ac.kr,mybae73@naver.com
}

\begin{abstract}
Today, as the supply of smart phones becomes active and a variety of functions of smart phones and services based on internet is utilized, a wide range of applications are being developed and deployed. Despite the rapid growth of smart phones and the application market, the attendance book which is currently being used is still in its primitive forms. Previously developed automatic attendance or absence check system requires additional equipments such as RFID reader and fingerprint reader, with higher deployment costs and has problems of management such as mechanical failure and dishonest attendance.

In this paper, an automatic attendance check system based on smart phones utilizing the $Q R$ code for recognizing the code using the built-in camera function of smart phones and accessing the information included in the recognized code in real-time basis is proposed. The proposed system, by utilizing the built in camerafunction of smart phones, can reduce the initial deployment costs because it does not require additional equipments when building the system and has partially solved the problems of dishonest attendance and improved the recognition rate.
\end{abstract}

Keywords: Automatie attendance gheck system, QR code, RFID, smart phone

\section{Introduction}

The smart phones unlikefeature phones can freely install and execute a variety of applications and especially, os the QR code scanner program was widely developed using the built-in camera function, the QR code has caught the attention as a market tool. Since QR code is an interactive code that connects the information of online and offline, when utilizing the QR code, a real time access to the information can be possible with a simple operation called the 'QR code scan' rather than accessing the website by entering the long and complicated ORL by the smart phone users $[1,2]$.

Despie the rapid growth of smart phones and the application market, in the case of attendance book which are currently being used by many schools and academies, it is still in its primitive forms. In the case of such attendance book, it has many problematic elements such loss of efficiency due to simple attendance management function, reduction in work efficiency due to extra computing process, waste of supplies due to creation of extra attendance book, etc.

According to the recent development of auto recognition technology such as RFID, label, card, etc, and the biometic technology such as fingerprint recognition, facial recognition, etc, the automated attendance and absence system that automatically manages the attendance and absence of students utilizing such technologies have advantages such as unmanned verification of attendance and absence, reduced verification time for attendance and the internet verification of attendance and absence. However, in order to structure the automation system, it has problems in terms of costs because it requires additional equipments such as 
RFID reader, fingerprint reader and clicker, etc, and has problems of management due to dishonest attendance by loaning the card and photos.

In this paper, an attendance and absence management system of students using the QR code that can store the data is proposed. The previous attendance and absence management system has an inconvenience of the professor directly entering the attendance status of the students in the corresponding classroom and has a disadvantage of losing time for lecture due to such verification. The proposed system operates in the following manner. First, in the case of students, after installing the application of the proposed system, by scanning the newly generated QR code for each classroom with their smart phones, the attendance is automatically recognized. Recognized information are stored in DBMS and whenever required by students, an information and time table on their attendance status can be viewed, managed and printed at any time using the web or the application. In the case of professors, the attendance status can be verified at any time through the web or the application depending on the requirement, and the information on students can be viefwed through the administrator mode. Since the proposed system does not require additional equipments, the system can be introduced with the least amount of costs, and because the attendance status can be checked using their smart phones, a quick and accurate attendance check is possible.

The configuration of this paper is as follows. In Chapter 2, we will look into the technology related to the QR code and introduce the technology of attendance and absence system previously developed. In Chapter 3, the new attendance and absence management system proposed in this paper will be designed and in Chapter 4, the implementation process of automated attendance and absence check system using the QR code is explained. Lastly in Chapter 5, it will describe the condlusion and future tasks.

\section{Related Works}

In this section, we will look into the technology related to the QR code and introduce the technology of attendance and absence system previously developed.

\subsection{QR Code}

QR code is a matrix type two dimensional bar code that shows the information in black and white plaid pattern. QR code is mainly used in Japan, Korea, United Kingdom and the United States and the name was derived from the registered trademark of Denso Wave called the 'Quick Response'. By overcoming the capacity limitations of conventional bar codes, the two dimensional bar code with expanded form and contents having the information of the length and breadth $1 t$ can store the data of characters in addition to numbers. Usually, it is utilized by reading if with a dedicated scanner or digital camera [3].

Copventional bar codes can store the number or text information in one-dimensional method whereas the QR codes can store much more information by having two dimensional form in horizontal and vertical directions in which it can store the character data such as alphabet and Chinese characters in addition to numbers. For the size of the QR code, the application version is determined according to the amount of data, type of character and error recovery label, and the cell is set according to the resolution of the printer or scanner. Version consists of 1-40 and each version has predetermined number of cells. Cell is a quadrangle point that forms the QR code. The cell starting with version 1 has 21 cells horizontally and 21 cells vertically, and 4 cells will increase for every increase in version, and in version 40, it is set to contain 177 cells by 177 cells. Figure 1 shows the structure of QR-code. 

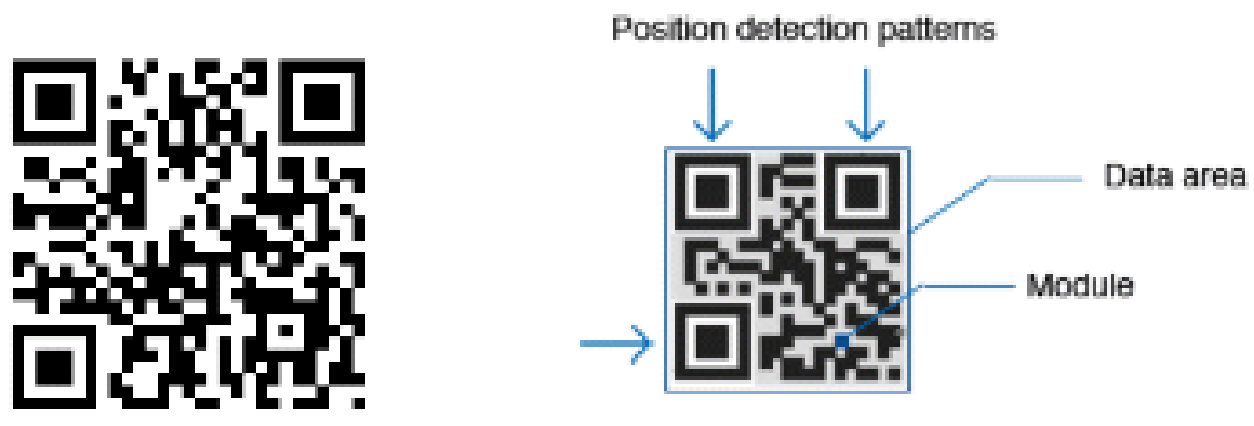

Figure 1. Structure of QR Code

In order to express and read data easily, QR-code is dividec into various areas including quiet zone, finder pattern, timing pattern, alignment/pattern, format information, version information and data and erro correction keys etc. QR-code can process all data including numbers, English characters, Chinese characters, Korean characters, symbols, Binary and control-code etc. Information quantities can be expressed up to 7,089 characters (numbers) with one eode.

Recently, iQR-code that enhanced QR code was developed. iQR-code can save more information quantities than the existingQR-code If it has the same size with $Q R$-code, it can save up to $80 \%$ increased, information quantities and if it has the same information quantity, it can make $30 \%$ decreased size information quantities. Additionally, iQR-code can generate smalter codes than the existing QR-code. While the initial composition of QR-code is 11 cells by 11 cells, iQR-code has 9 cells by 9 cells composition and the code arealcan be made up to $60 \%$ smaller than the existing QR-code [2].

With $\mathrm{iQR}$-code, we can set a higher restoration capability than with the traditional QR-code. The error correction level is up to $30 \%$ of the entire code for the QR-code, meaning it is possible to restore the code with this percentage of smeared or damaged area. On the other hand, this is increased to $50 \%$ with the iQR-code.

\subsection{Existing Attendance Management System}

The attendarce and absence management of the traditional way have used the method of directly entering the attendance status of the students for each classroom in hand writing with an inconvenience of creating a separate attendance book for each corresponding classes. In the case of initial electronic attendance and absence system introduced to implement such inconvenience, the teacher had to enter the hand written details for each classroom into the attendance and absence management system to view the attendance or absence status or to print the attendance book, but it was a hassle that required a double work.

Recently, due to the development of auto recognition technology such as RFID, label, card, etc, and the biometic technology such as fingerprint recognition, facial recognition, etc, an automated attendance and absence management system that automatically manages the attendance and absence of students utilizing such technologies are widely studied and developed.

RFID based automated attendance and absence system [4] is a system that automatically aggregates the attendance and absence status when the student's smart card attached with RFID tag is recognized by the reader installed in the entrance and it provides efficient 
attendance and absence management compared to the previous attendance book which was hand written. However, the system is expensive to build and includes problems such as the possibility of loaning the smart card, stealing, dishonest attendance, and disadvantage of attendance not being recognized when the smart card is lost or not in possession and doing the attendance check and leaving the classroom without actually attending the class.

An automated attendance and absence system [5] based on the fingerprint is a system that aggregate the attendance and absence status by recognizing the fingerprint of students using the fingerprint reader is a trustworthy method that can uniquely identify the person without the problem of being lost, loaned or stolen, but like the RFID based system, it has a disadvantage of being expensive to build. In the student attendance and absence management system using the self-organized type of facial recognition, in order to improve on the point of verifying the actual card holder in the previous smart card based electronic attendance and absence system, a client-server system that automatically manages the attentance status of the corresponding course by recognizing the person's facial information using the selforganization neural network was developed [6]. As a result of testing the system in the CS environment in order to test the developed system, it has shown more than $92 \%$ effectiveness.

The clicker [7] is a two-way wireless lecturing system consisting of student responder in the size of remote control and the receiver attached to the computer and it is a useful lecturing support system that enables effective questions and answers between teachers and students as well as automatically checking the attendance and absence. However, the clicker includes cost-occurring problems due to the clicker being a separate terminal which must be provided equally with the number of students and operational problems due to the method of being managed by loaning the clicker after the mass purchase by the college.

Such automated attendance and absence system reduces the time of checking the attendance and absence due to unmanned operation and has the advantage of being able to check the attendance status by themselyes over the Internet. However, in order to structure the automated system, there is a costly problem on the installation cost for introducing the system because it requires separate equipnifents such as RFID reader, fingerprint reader, clickers, etc. In addition, there could be problems such as not being recognized of attendance when the smart card is lost or not in possession, loaning of cards or dishonest attendance using stolen cards and a difficult situation of checking the attendance and absence due to equipment failure such as reader efror and damaged cards [8].

In this paper, an automated attendance check system that improves and supplements the disadvantages of existing systems is proposed. The automated attendance check system developed in thís paper is OTIP (On Time In Place) system.

\section{Design of OTIP System}

The biggest problem of automated attendance and absence system is the costly problems of building the system, methods of resolving the dishonest attendance and equipment malfunction.

The characteristics of the system proposed in this paper is a method of recognizing the attendance by utilizing the QR code where the attendance is automatically processed by recognizing the generated QR code using the smart phone of the student after installing the application in the student's smart phones. This does not require additional purchase of readers for processing the attendance check and has no installation cost, and because the attendance is recognized using their own phones, the possibility of dishonest attendance is very low.

Figure 2 shows flowchart of the proposed automatic attendance check system in this paper. 


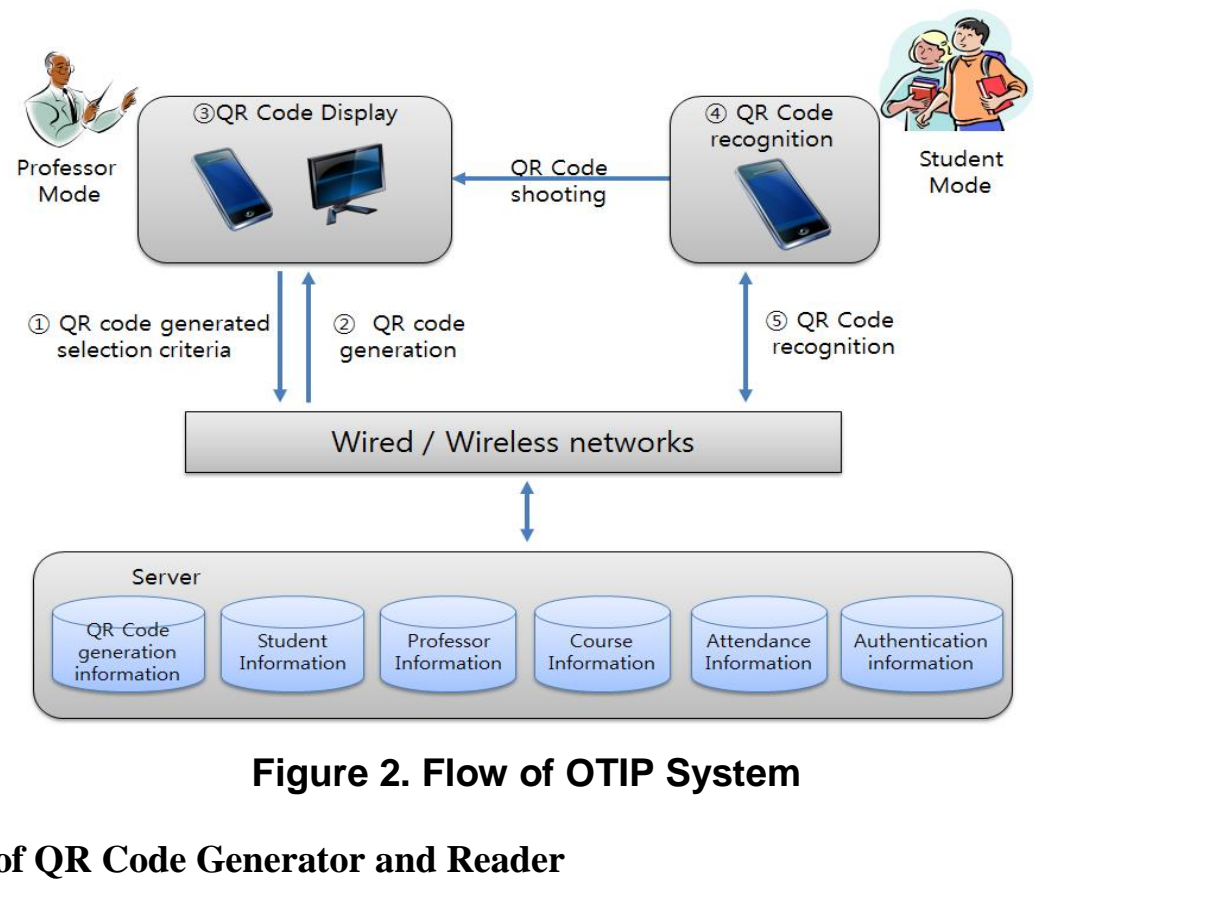

3.1. Design of QR Code Generator and Reader

QR code generator and readers are widely developed and used. However, previously developed $\mathrm{QR}$ code generator andreaders cannot automatically insert the information required by the automated attendance and absence system proposed in this paper into the $\mathrm{QR}$ code, and in addition, the contents of the QR code is not encrypted so it has the risk of information leakage.

The QR code generator of the proposed system generates the QR code in the server that corresponds to each course. In the QR code generated at such time, it stores information on the course necessary for the attendance check (title of the course, date, start/end time, etc.), the start time and end time of QR code recognition and the generating time of the QR code. Especially, in order to prevent the reuse of the QR code, after a certain time, it generates a new QR code. In addition, the information inserted in the QR code will use the seed encrypted algorithm for (encryption where unless a dedicated reader is used, the contents of the QR code cannot be known. The generation of the QR code is made on the server by the teacher and the teacher can select the media to express the generated QR code such as smart phones, printer monitor, etc.

The QR code reader sends the obtained information and the personal identification to the server by obtaining the information inserted in the $\mathrm{QR}$ code after the QR code generated by the QR code generator is recognized. The server renews the database after processing the transmitted data and after updating the data to meet the situation of the teacher and student, it renews the screen of each user.

\subsection{Design of Database for Attendance Management System (OTIP)}

The database structure for configuring the system consists of Certification_Tb, Professor_Tb, Student_Tb, Class_Tb, QR code_Tb, Attendance_Tb.

First, Certification_Tb is a table for authenticating whether the user is valid or whether the person accessing is a student or a teacher, and when the program is executed in order to use the proposed system, it has an advantage of not having to log in each time once the authentication is received the first time. 
Class_Tb is a table that contains the information on the course, students and teachers, and in the case of Professor_Tb, it is a table that stores the information of teachers who uses the server program, title of the course and the attendance status, and the Student_Tb is a table that includes student's information and used as the basic data for listing the student information in the Class_Tb.

QRcode_Tb is a table for setting the attendance time of each class, selecting the method of attendance check and the start time and end time of QR code recognition, and the server generates the QR code based on this table.

The attendance and absence table is a table for storing the attendance status, QR code generated numbers and information related to the attendance and absence, and based onothe extracted information from this table, the attendance book for each course can be created.

\section{Development of OTIP System using QR Code}

The system proposed in this paper is implemented with seryer related program, teacher's application program and the student's application program Linux-based apache server was used for the server related program, My-Sql was used for the database/and PHP was used to create the web program.

Especially, the QR code is generated in the seryer upon request from the teacher and for security, the seed encrypted algorithm was used for encryptionand inserted into the QR code. For the application program, android based teacher's mode and student's mode was separately developed using the eclipse, and in the teacher's mode, the information on the QR code generation and attendance status oan be checked and in the student's mode, QR code recognition and attendance stats can be checked.

\subsection{Application Program for Professor}

The configuration of teacher's main Screen is show in Figure 3, and consists of user authentication, QR code attendance check, teacher's dedicated menu, help and program termination. It is used after obtaining the authentication through user authentication. Especially, when authenticated through the user authentication, it eliminates the inconvenience of logging in each time.

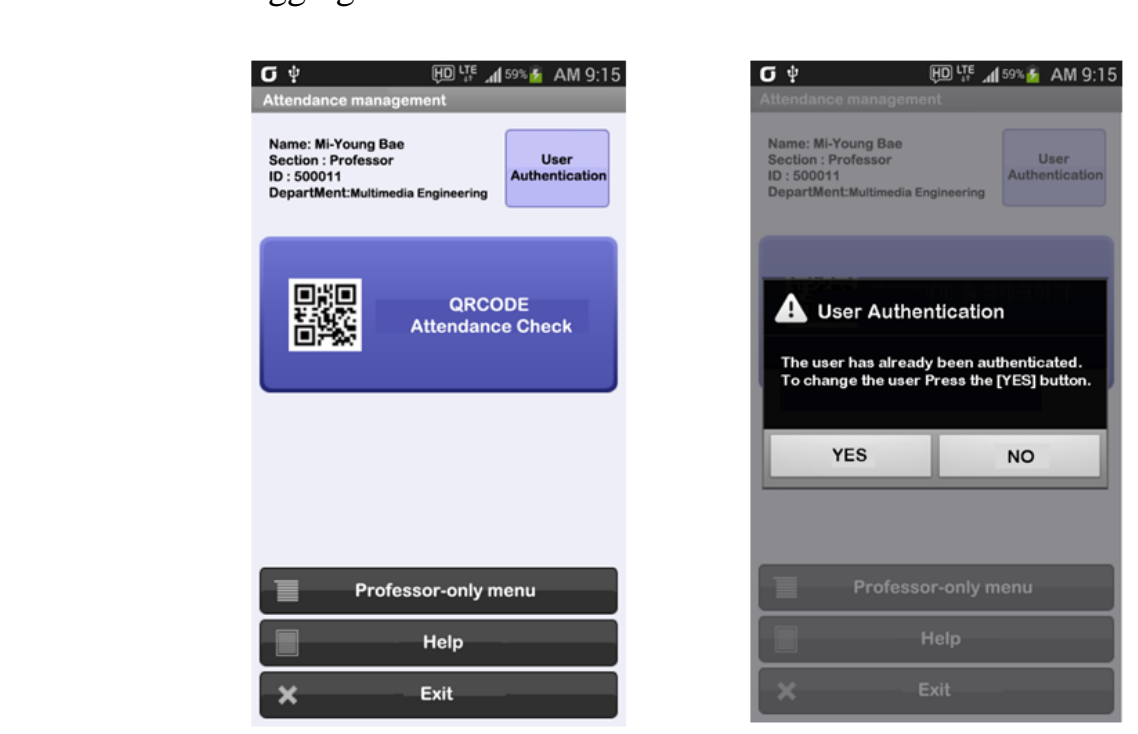

Figure 3. Professor Mode of OTIP System 
After being authenticated, when selecting the QR code attendance check button among the teacher's dedicated menu, courses in charge by the teacher will appear and after selecting the course desired for checking the attendance, in the screen indicated with detailed time of the class, by selecting the item that correspond to the class time, the QR code inserted with the course information is generated like Figure 4.
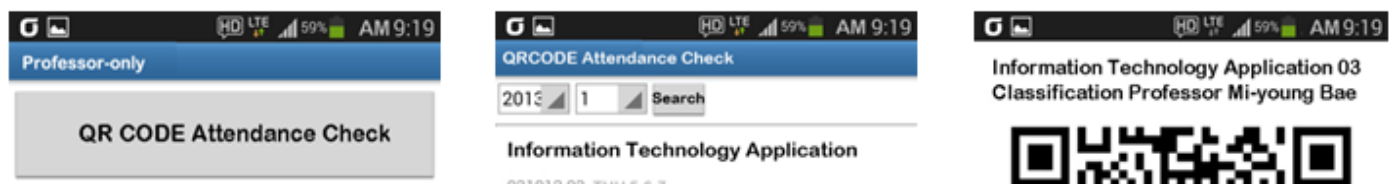

Information Technology Application

03101203 THU 5,6,7.

Information Technology Application

03101206 TUE 5,6,7

C Language

60510101 THU 1,2 FRI 1,2

C Language

60510102 THU 3,4 FRI 3,4

Figure 4. QR Code Generation for Attendance Check

In order to prevent the dishonest atrendance due to reuse of students, initially generated QR code will disappear after a certain amount of time and the new QR code is generated.

\subsection{Application Program for Students}

The configuration of the main menu screen of the student's application is shown in Figure 5 which consists of user authentication, QR code attendance check, viewing the attendance check details, hetp and program ternination.
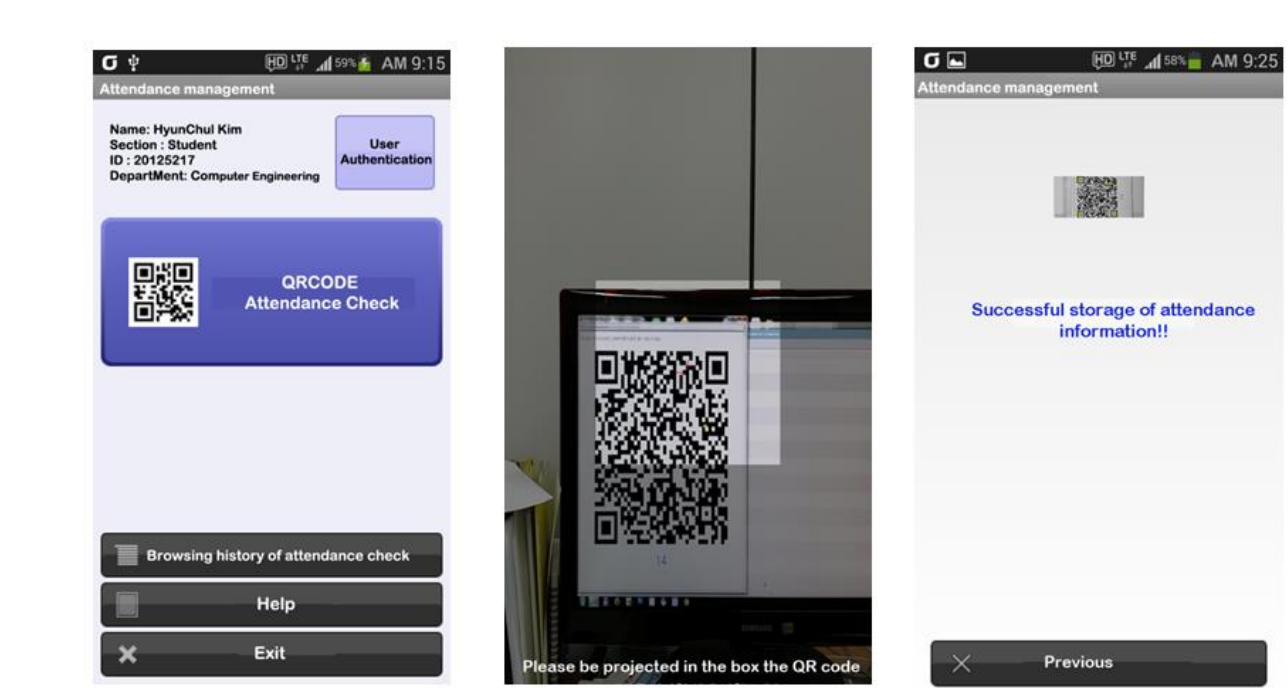

Figure 5. Student Mode of OTIP System

Like the teacher's application program, the authentication is obtained through user authentication so it has no inconvenience of logging in each time. When checking the QR 
code attendance in the student's application, the QR code reader is immediately executed and such time, by scanning the QR code generated by the teacher, the attendance check is made. In addition, when the history of attendance check is selected, the attendance status of all courses attended by the student can be checked in real-time basis.

\section{Conclusion}

The previous attendance system has problems such as dishonest attendance by loaning the student card equipped with RFID chip to their friends, recognition rate of the system, inability to recognize due to damaged magnetic cards, and has increase in costs of building the system due to the installation of card readers and issuance of cards.

In this paper, in order to improve on such problems, an automated attendance and absence check system using the QR code was proposed. The proposed system does not require a separate purchase of the QR code generator for the attendance check. In/addition, it does not require a separate purchase of the QR code reader to recognize the generated QR code. Since teachers and the students can download the application developed in the proposed system using their smart phones to be utilized as the code gererator and the reader, there is no incurring of costs for purchasing the equipments to be used for generating or reading, thus it is economical since it does not increase the installation costs when building the system. In the proposed system, in order to solve the problem of dishonest attendance, the QR code is regenerated every 15 seconds.

QR code has superior recognition rate than the prevtous bar code where it can still be recognized even if parts of the code 8 gontaminated or damaged, so it will reduce the case of not being able to recognize due to the damage of code.

The goal of the future study is to improve the proposed system so that it can be applied to any situation and be able to link with a varety of academic management system.

\section{Acknowledgements}

The research was supported byca grant from 2013 LINC (Leaders in Industry-University Cooperation) Progam of Andong National University.

\section{References}

[1] K. Lee, "Implementation of Smartphone System using QR Code in Mobile Environment", IJSEIA, vol. 7, no. 6, (2013), pp.137-146.

[2] X. Yu, Y Song, Y. Yu, S. Yu, H. Cheng and Y. Guan, “An Encryption Method for QR Code Image Based on ECA" USIA, vol. 7, no. 5, (2013), pp. 397-406.

[3] bttp://www.qrcode.com/

[4] S. H. Park and B.-C. Moon, "The Development of Attendance Management System Using the RFID", Jounhal of KAIE, vol. 11, no. 2, (2007), pp. 139-146.

[5] J. Ramakrishnan and M. Ramakrishnan, "An Efficient Automatic Attendance System Using Fingerprint Reconstruction Technique”, Int. J. Comput. Sci. Inf. Sec., vol. 10, no. 3, (2012), pp. 77-82.

[6] W. B. Lee, "An Attendance-Absence Checking System using the Self-organizing Face Recognition”, Journal of the Korea Contents Association, vol. 10, no. 3, (2010), pp. 72-79.

[7] D. Duncan, "Clickers: A New Teaching Aid with Exceptional Promise", Astronomy Education Review, vol. 5, no. 1, (2006), pp.70-88.

[8] J.-I. Choi, D.-S. Yoon, J.-H. Chang, “A System for Marking the Absence using a Smart-Phone”, Journal of the Korea Academia-Industrial Cooperation Society, vol. 12, no. 9, (2011), pp. 4160-4168. 


\section{Authors}

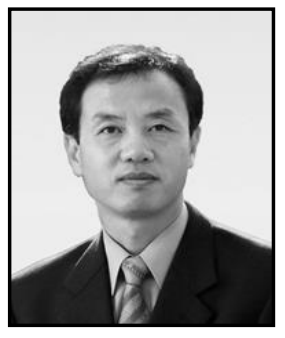

Dae-Jea Cho, $\mathrm{He}$ received the Ph.D. degree in computer engineering from Kyungpook National University, Korea, 2001. He is professor at Andong National University, Korea. His areas of interest include digital watermarking and multimedia contents authentication.

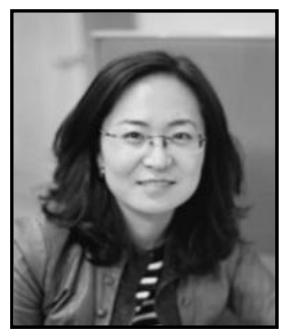

Mi-Young Bae, She received the B.S. degree in computer engineering from Andong National University, Korea, 1996, and M.S. degree in computer engineermg from Andong National University, 2000. She is studying Ph.D. Course in Information communication engineering from Andong National University, 2012. Her areas of interest include computer vision and mobile programming.

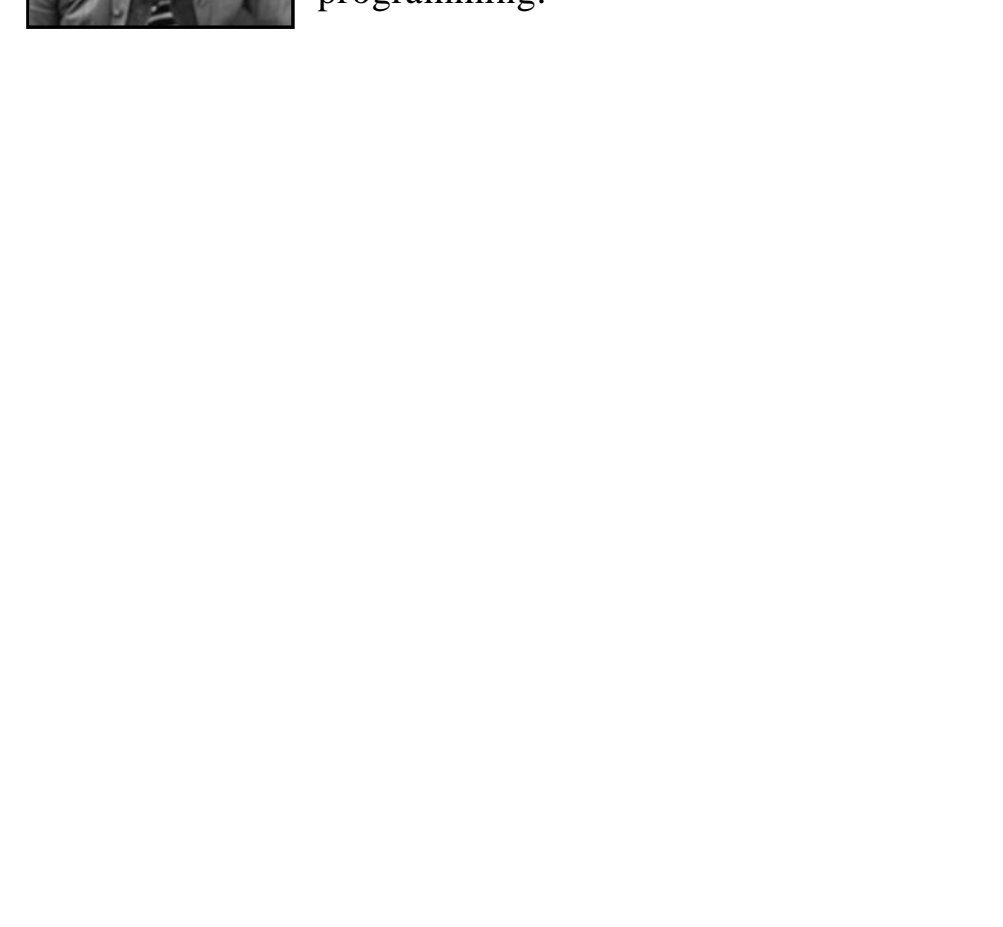


International Journal of Multimedia and Ubiquitous Engineering Vol. 9, No. 10 (2014)

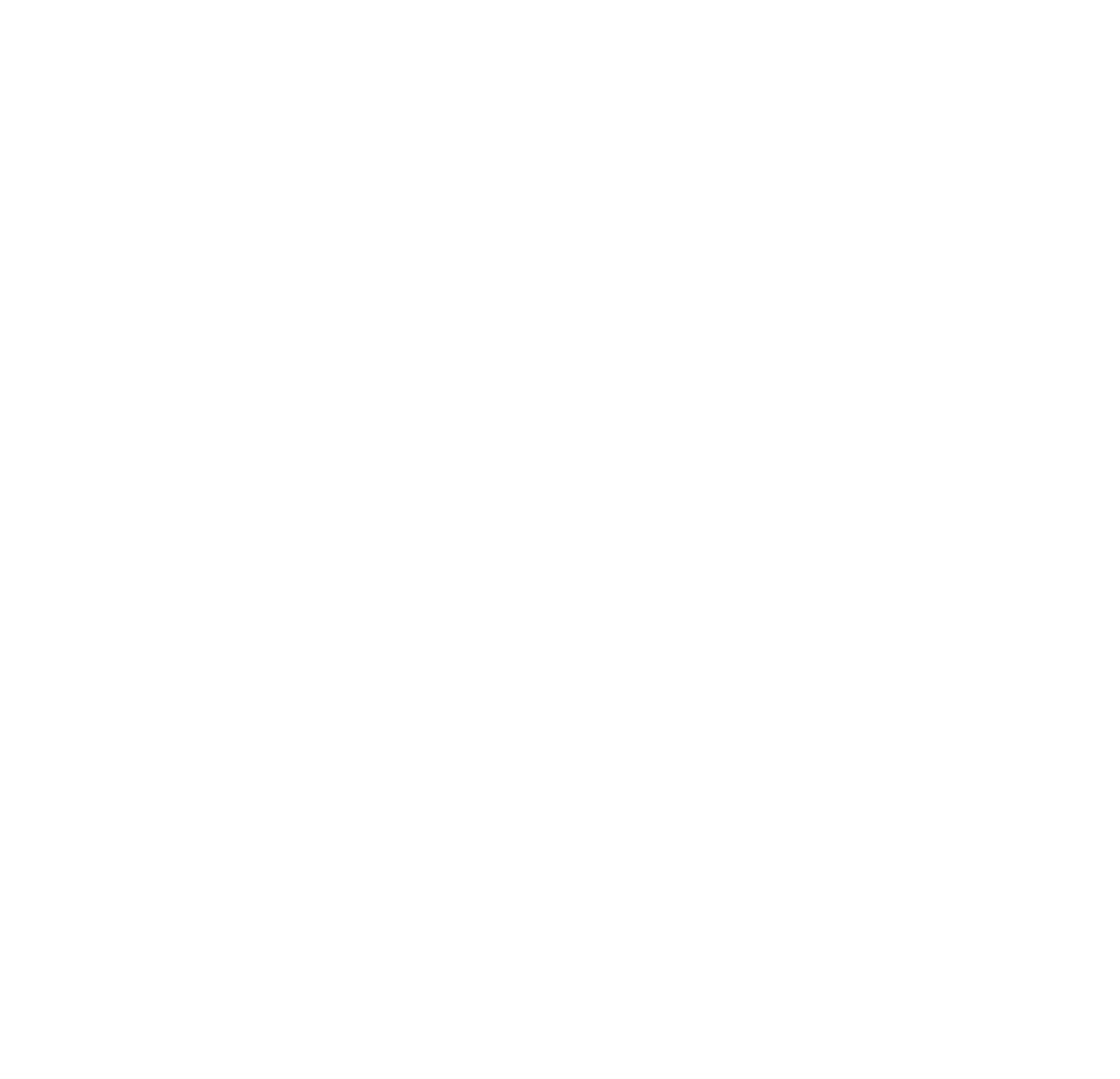

